\title{
Selectivity in the reinnervation of the lateral gastrocnemius muscle after nerve repair with ethyl cyanoacrylate in the rat
}

\author{
Thomas Landegren $^{1}$ *, Mårten Risling ${ }^{2}$, Henrik Hammarberg ${ }^{1}$ and Jonas K. E. Persson ${ }^{3}$ \\ 1 Section of Hand Surgery, Department of Clinical Science and Education, Södersjukhuset, Karolinska Institutet, Stockholm, Sweden \\ 2 Retzius Laboratory, Experimental Traumatology Unit, Department of Neuroscience, Karolinska Institutet, Stockholm, Sweden \\ ${ }^{3}$ Department of Clinical Neuroscience, Karolinska Institutet, Karolinska Universitetssjukhuset Solna, Stockholm, Sweden
}

\section{Edited by:}

Mattias Skšld, Uppsala University,

Sweden

Reviewed by:

Ching-Hsiang Wu, National Defense

Medical Center, Taiwan

Eng-Ang Ling, National University of

Singapore, Singapore

*Correspondence:

Thomas Landegren, Section of Hand

Surgery, Department of Clinical

Science and Education,

Södersjukhuset, Karolinska Institutet

SE-118 83 Södersjukhuset,

Stockholm, Sweden.

e-mail: thomas.landegren@ki.se
There is a need for complementary surgical techniques that enable rapid and reliable primary repair of transected nerves. Previous studies after peripheral nerve transection and repair with synthetic adhesives have demonstrated regeneration to an extent comparable to that of conventional techniques. The aim of this study was to compare two different repair techniques on the selectivity of muscle reinnervation after repair and completed regeneration. We used the cholera toxin $B$ technique of retrograde axonal tracing to evaluate the morphology, the number, and the three-dimensional location of $\alpha$-motoneurons innervating the lateral gastrocnemius muscle and compared the results after repair with either ethyl cyanoacrylate (ECA) or epineural sutures of the transected parent sciatic nerve. In addition, we recorded the wet weight of the muscle. Six months after transection and repair of the sciatic nerve, the redistribution of the motoneuron pool was markedly disorganized, the motoneurons had apparently increased in number, and they were scattered throughout a larger volume of the spinal cord gray matter with a decrease in the synaptic coverage compared to controls. A reduction in muscle weight was observed as well. No difference in morphometric variables or muscle weight between the two repair methods could be detected. We conclude that the selectivity of motor reinnervation following sciatic nerve transection and subsequent repair with ECA is comparable to that following conventional micro suturing.

Keywords: nerve repair, cyanoacrylate, synthetic adhesive, retrograde tracing, gastrocnemius muscle, spinal misdirection, sciatic nerve, peripheral nerve

\section{INTRODUCTION}

Experimental and clinical results of neural anastomosis using microsutures have been shown to be less than satisfactory in adults (Sullivan, 1985; Vertruyen et al., 1994; Siemionow and Brzezicki, 2009). Nevertheless, the repair technique using interrupted nylon microsutures is currently accepted as the golden standard of peripheral nerve repair (Millesi, 1973; Dvali and Mackinnon, 2007). Moreover, this technique with repeated tissue handling and consequent trauma has been shown to injure the nerve tissue, hinder the sprouting of axons, and compress the blood supply to the fascicles. This may hamper the growth of regenerating axons and ultimately impede complete nerve function recovery (Brushart et al., 1983; Bertelli and Mira, 1993; Suri et al., 2002).

To improve the functional outcome of peripheral nerve repair, a sutureless seam with synthetic adhesive has been proposed as an option to microsutures for achieving proper coaptation of the nerve endings. Data from previously published experimental reports have demonstrated recovery after repair of transected peripheral nerves using cyanoacrylate (CA) to an extent comparable to that after conventional microsurgical suturing (Choi et al., 2004; Pineros-Fernandez et al., 2005; Landegren et al., 2006).

However, irrespective of the operative method, functional restoration following nerve injury and subsequent repair is often disappointing. The type of injury, injury location, time delay from injury to surgery, age, and physical condition of the individual are factors that have been suggested to influence the results of nerve regeneration (Fu and Gordon, 1995a,b; Verdu et al., 2000). Misdirection of regenerating axons is also a factor that may explain poor functional recovery. When reinnervation of a repaired motor nerve has been completed, misdirected reinnervation of the target muscle can lead to involuntary muscular movements accompanying voluntary movements (e.g., synkinesis; Fu and Gordon, 1995a,b; Sumner, 1990). After reinnervation of a sensory nerve, misdirection may similarly result in persistent sensory dysfunction (Galtrey and Fawcett, 2007).

Different retrograde tracing techniques, including singlelabeling, have been used to investigate the accuracy of motor and sensory nerve regeneration after experimental nerve repair. The results of these studies all suggest that there is a preferential reinnervation of the original target muscle (Brushart, 1993), but the specificity of reinnervation is limited (Zhao et al., 1992). However, little is known about the effect of applying synthetic adhesive on motoneuron regeneration specificity.

Since sensory and motor Schwann cell phenotypes differ in their patterns of trophic factor expression, it seems possible that this circumstance could help regrowing axons to find proper motor or sensory fascicles in the distal stump (Hoke et al., 2006). Adding synthetic materials to the lesioned nerve endings might possibly 
exert a negative influence on the recognition of such trophic guidance cues. In this study we have assessed whether nerve repair with CA would impair the precision in reinnervation, compared to conventional nerve suturing.

The aim of the present study was to compare two different repair techniques: micro suturing versus coaptation with ethyl cyanoacrylate (ECA) in the process of reinnervating the lateral gastrocnemius muscle (LGC) following sciatic nerve transection and subsequent repair. After reinnervation had been completed, retrograde neuronal tracing with single- and double-labeling techniques was performed to evaluate quantitatively and descriptively the accuracy of motor axons for regeneration to the original target. In addition, the weight of the lateral LGC was determined at the end of the experiment, and the results were correlated with those obtained after retrograde axonal labeling.

\section{MATERIALS AND METHODS ANIMALS}

Eighteen female Sprague-Dawley rats weighing 180-200 g and obtained from B \& K Universal, Sollentuna, Sweden, were used in the experiments. The animals were caged in small groups and their environment was maintained at room temperature with 12h light-dark, day-night cycles. They had free access to standard rodent food and water. All experiments were carried out with the approval of the Ethics Committee for Animal Research in Southern Stockholm.

\section{SURGICAL PROCEDURES AND POSTOPERATIVE CARE}

Animals were anesthetized by injection of Hypnorm vet ${ }^{\circledR}$ (fentanyl $0.05 \mathrm{mg} / \mathrm{ml}$ and fluanisone $2.50 \mathrm{mg} / \mathrm{ml}$, Janssen Animal Health Ltd) $0.4 \mathrm{ml} / \mathrm{kg}$ intraperitoneally and midazolam $2 \mathrm{mg} / \mathrm{kg}$ intraperitoneally. One surgeon did all the operations using an aseptic technique and microsurgical dissection under an operating stereomicroscope (ZEISS OPMI-9, Carl Zeiss, Göttingen, Germany). The animals were kept on a thermostat-adjusted heating pad to maintain normal body temperature during all procedures. The left sciatic nerve of each rat was exposed through a dorsal gluteal-splitting approach between the gluteal muscle and the femoral biceps muscle. The nerve was transected at the midthigh level and repaired immediately by direct coaptation of the nerve ends with ECA $(n=10)$ and epineural sutures $(n=10)$, respectively. The ECA used in this study is commercially available (Evobond $^{\circledR}$, Tong Shen Enterprise Co., Ltd, Taiwan) and supplied in a 2-ml plastic ampoule with a stiff-tip applicator at one end. In this configuration, it is impossible to apply the adhesive during a microsurgical procedure. For this reason, a couple of drops $(0.2 \mathrm{ml})$ of the adhesive were transferred into a $1-\mathrm{ml}$ syringe with a needle. After transection of the nerve, a minimal amount was applied gently to the proximal nerve ending which had just been dried to promote adhesion. Sealing was facilitated by a sterile sheet of absorbable gelatin sponge, $1 \mathrm{~mm}^{2}$, placed under the nerve to avoid the spontaneous retraction of the proximal and distal segments of the nerve. The nerve endings were then carefully brought together microsurgically with two pairs of microforceps, after which the gelatin sponge was removed. On application, the liquid monomer formulation polymerises instantly to a thin polymer film that adheres to the two opposite nerve endings. Any surplus adhesive was removed. A single 9/0 monofilament nylon suture (Ethicon, J \& J, Somerville, NJ, USA) marked the anastomotic site in the epineurium of the proximal nerve ending. The overlying muscles and skin were closed in layers with single 5/0 monofilament nylon sutures (Ethicon, Somerville, NJ, USA). Other rats had their transected nerve repaired with three $9 / 0$ monofilament interrupted nylon sutures (Ethicon, J \& J, Somerville, NJ, USA) evenly spaced in the epineurium and followed by skin closure. Buprenorphine hydrochloride (Schering-Plough, Kenilworth, NJ, USA) was administered subcutaneously $30 \mathrm{~min}$ after the repair for prevention of pain. The rats in the two groups recovered from the anesthetic and were allowed to move freely immediately after the operation.

Three months after the repair, four rats in each group were chosen at random and reanesthetized. The anastomosis in the sciatic nerve was explored and examined under the operating stereomicroscope. The tissues were then closed and the animals were allowed to survive until the end of the predetermined postoperative period.

\section{TRACING OF LGC NEURONS}

Six months postoperatively, the animals were reanesthetized and the sciatic nerve and tibial branch of the LGC were exposed bilaterally. The tibial branch of the left LGC was identified and cut at a location just before it entered the muscle. The proximal nerve ending was inserted into a polyethylene capsule containing a solution of $1 \%$ cholera toxin subunit B low salt (CTB; List Biological Laboratories, Campbell, CA, USA) and left in place for $30 \mathrm{~min}$. The capsule was then removed, and the surrounding area was rinsed with saline. Subsequently, the tibial branch of the opposite LGC at the corresponding site was processed by the same method and used as a control.

Three days later the animals were deeply reanesthetized and perfused via the ascending aorta with $200 \mathrm{ml}$ saline at body temperature, followed by $700 \mathrm{ml}$ of ice-cold $4 \%(\mathrm{w} / \mathrm{v})$ paraformaldehyde in a $0.15-\mathrm{M}$ phosphate buffer $(\mathrm{pH} 7.2-7.4)$. The spinal cord was exposed and the exit levels of the lumbar spinal roots were used to identify the individual lumbar spinal cord segments. The L5 spinal cord segment was carefully removed and the left side was marked by a longitudinal shallow groove. The L 5 spinal cord segment was post-fixed for $4 \mathrm{~h}$ at $+4^{\circ} \mathrm{C}$ and cryoprotected in sucrose $(30 \%$ in $0.01 \mathrm{M} \mathrm{PB}, \mathrm{pH} 7.3)$ at $+4^{\circ} \mathrm{C}$ overnight.

Frozen cross-sections were cut at $14 \mu \mathrm{m}$ with a Cryostat (Micram HM 560, Heidelberg, Germany) and every fifth section was thaw-mounted serially in a rostro-caudal sequence on gelatincoated slides and prepared for the single-labeling process. Remaining sections were thaw-mounted serially in the same manner and stored for the double-labeling process. This resulted in neighboring sections on each slide taken from the L5 spinal cord segment at an interval of $70 \mu \mathrm{m}$.

\section{IMMUNOHISTOCHEMISTRY}

For the detection of $\mathrm{CTB}$, the sections were preincubated with $5 \%$ bovine serum albumin and $0.3 \%$ Triton X-100 in $0.01 \mathrm{M}$ PBS ( $\mathrm{pH} \mathrm{7.4)} \mathrm{at} \mathrm{room} \mathrm{temperature} \mathrm{for} 30 \mathrm{~min}$ (all primary and secondary antisera were diluted in this solution) and then incubated in a humid atmosphere with goat anti-CTB (List Biological 
Laboratories, Campbell, CA, USA, dilution 1:1000) overnight at $4^{\circ} \mathrm{C}$. Thereafter, the sections were rinsed in PBS $(2 \times 10 \mathrm{~min})$ and incubated with Cy3-conjugated donkey anti-goat IgG (Jackson ImmunoResearch, PA, USA, dilution 1:1000) for $1 \mathrm{~h}$ at room temperature, followed by rinsing in PBS $(2 \times 10 \mathrm{~min})$. Single-labeling sections were then mounted in a 1:3 solution of glycerol and PBS.

In the cases of double-labeling, sections were labeled using two different fluorophores and processed for CTB immunohistochemistry as described, combined with immunoprocessing with one of the two following cellular markers: rabbit anti-CGRP (Bachem, Bubendorf, Switzerland, dilution 1:400), mouse antisynaptophysin (Sigma, Saint Louis, MO, USA, dilution 1:400). After rinsing with PBS $(2 \times 10 \mathrm{~min})$, the sections were incubated with Cy2-conjugated donkey anti rabbit IgG (Jackson ImmunoResearch, PA, USA, dilution 1:200) or donkey anti-mouse IgG (Jackson ImmunoResearch, PA, USA, dilution 1:200) for $1 \mathrm{~h}$ at room temperature. Subsequently, the sections were rinsed in PBS $(2 \times 10 \mathrm{~min})$ and mounted in a 1:3 solution of glycerol and PBS.

\section{IMAGING}

The sections were examined using a fluorescence microscope and appropriate filter combinations for the fluorophores used (Nikon Eclipse E600, Nikon, Tokyo, Japan). Fluorescence microscopic images were captured with a digital camera (Nikon Digital Sight DS-U1), whereas confocal microscopic images were processed in software and digitalized (described later). Identical magnification was used for all the micrographs for each imaging technique.

The micrographs were imported into and edited in Adobe ${ }^{\circledR}$ Photoshop $^{\circledR}$ CS software (version 7.0; Adobe Systems Inc., San Jose, CA, USA). The only digital manipulations done were limited contrast and/or brightness enhancement to allow adequate comparison of images and, if the size of a region of interest was too big to be captured in one frame, a series of adjacent overlapping frames was shot under the same conditions and montaged together. Retrogradely labeled motoneurons (RLMs), with a diameter of $\geq 30 \mu \mathrm{m}$, a clearly visible nucleus and polygonal in shape, were counted in order to selectively estimate the labeled population of $\alpha$-motoneurons ( $\alpha$-MNs) and avoid inclusion of $\gamma$-motoneurons. Analyses, quantitative or descriptive, were performed bilaterally using digitalized images displayed on a computer monitor. Quantitative comparisons of the two repair methods were made as a percentage of the operated side proportionately to the unoperated control side (mean values, expressed as percentages, for the two repair methods were determined) unless otherwise indicated. All images were masked and, to avoid bias, the observer was blinded to the method of repair.

\section{MORPHOLOGICAL EVALUATION}

At the end of the predetermined postoperative period ( 6 months), immunofluorescently labeled $\alpha$-MNs were evaluated with respect to nucleus and cell body shape, fluorescence intensity and neurite appearance.

\section{QUANTITATIVE AND DESCRIPTIVE ESTIMATIONS OF RETROGRADELY LABELED MOTONEURON}

To investigate the anatomical distribution of $\alpha$-MNs innervating the LGC in the lateral ventral horn of the spinal cord, micrographs from every five of all serially cut $14-\mu \mathrm{m}$ sections from the L5 spinal cord segment were transformed into separate layers using Adobe Photoshop 7.0. For each separate layer, the localization of every labeled $\alpha-\mathrm{MN}$ was selected manually, using a computer mouse, and marked with a dot. The demarcation of the gray matter was also outlined in these layers. Micrograph layers representing L5 spinal cord sections, with the exception of dots and lines of anatomical boundaries, were then concealed and stacks of serially superimposed layers representing RLMs (and the outlined gray matter boundaries) only were reconstructed to form a twodimensional digitized image. The transversal extensions of the RLM population, located in the lateral ventral horn of both sides, were then approximated to ellipses. The elliptical area was then measured using morphometric software (ImageJ 1.33u, National Institutes of Health, USA); see Figure 1. The distribution area of selected labeled neurons from the control side, which had been described as an ellipse, was delimited in a separate layer, reversed and superimposed as a template on the repaired side (dashed line) to facilitate estimations of the number of motoneurons located within or outside this assumed normal myotopic distribution area (Figure 1). The number of RLMs, identified by their localization in the lateral ventral horn of the L5 spinal cord sections, was determined bilaterally by manual counting in all serially cut $14-\mu \mathrm{m}$ sections. Correlations between repaired and control sides, followed by quantitative comparisons between the two repair methods, were worked out for RLMs in total and for RLMs within the assumed normal myotopic distribution area (described above), respectively. From each specimen, five RLMs were randomly selected from the repair and control sides, respectively, for cross-sectional cell body area measurements. RLMs were identified as previously described. By assuming that RLMs were elliptical in the transversal section, the areas were calculated by using the morphometric software ImageJ, NIH.

\section{DOUBLE-LABELING}

Cy3-conjugated profiles (CTB-positive motoneurons) were counted as RLMs innervating LGCs, whereas Cy2-conjugated profiles (CGRP-positive motoneurons) were counted as indefinable sciatic nerve-mediated neurons in the lateral ventral horn (Figure 2). Three sections were randomly selected from each specimen and their content of $\alpha$-MNs was counted bilaterally. The correlation between the number of RLMs and CGRP-labeled motoneurons was determined, whereupon quantitative comparisons between the two repair methods were made as described above.

\section{CONFOCAL MICROSCOPY AND ANALYSIS}

We used confocal microscopy to determine whether axons were synapsing on RLMs in the L5 spinal cord 6 months after sciatic nerve repair. Synaptophysin-immunoreactive (ir) profiles in the immediate vicinity of the cell bodies and proximal dendrites of RLMs were assessed quantitatively. From each repair method, six animals and five sections from each animal were randomly selected. Care was taken to choose all CTB-labeled $\alpha$ MNs (described above) in the lateral ventral horn from the two opposite sides with the aid of a fluorescence microscope. The morphometric examinations were optimized, with a minimum 


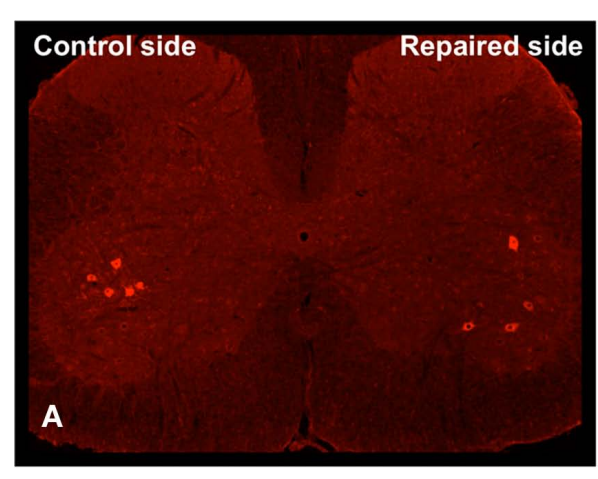

FIGURE 1 | Micrograph from a single separate transverse L5 spinal cord section showing RLMs bilaterally to the lateral gastrocnemius muscle (LGC) in the lateral ventral horn 6 months after transection and repair of

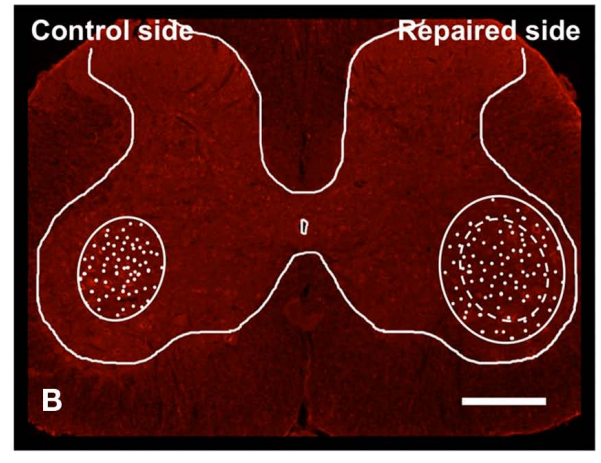

the left rat sciatic nerve with ETC (A). The background image (B) has been manipulated in Photoshop to remove RLMs and artifacts. Scale bar $=500 \mu \mathrm{m}$

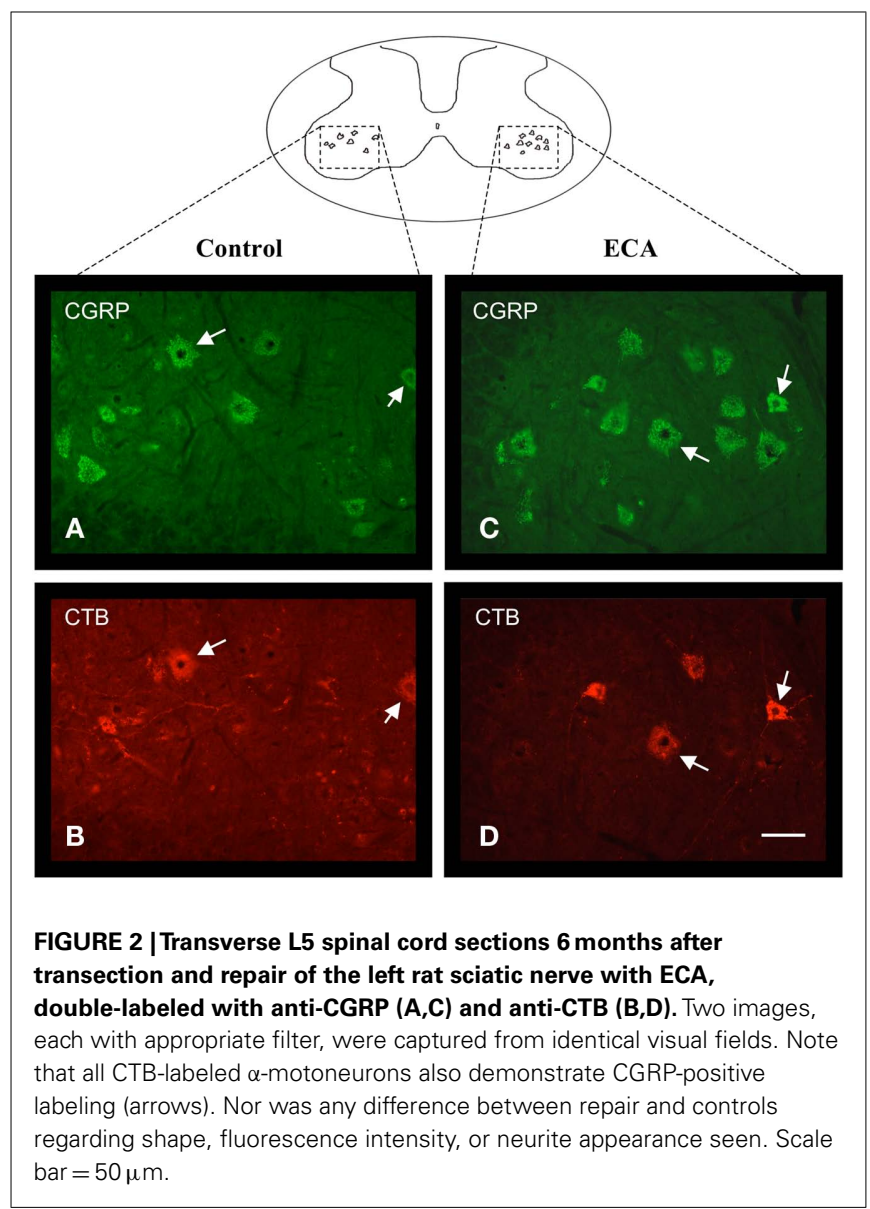

source of error, by reproducing each motoneuron in the microscope equipped with an e-Cl confocal system with an Argon Ion Laser $488 \mathrm{~nm}(40 \mathrm{~mW})$ and a HeNe Laser $543 \mathrm{~nm}(1.0 \mathrm{~mW})$. Fluorescence was filtered through a $500-530 \mathrm{~nm}$ band pass filter (green) and a 570-nm-long pass filter (red). Images were collected using an oil immersion $(60 \times)$ Plan Apo objective. Stacks of three optical serial confocal sections taken at $0.15-\mu \mathrm{m}$ intervals were reconstructed to produce two-dimensional digitized images using the volume rendering maximum paradigm in the EZ-C1 acquisition and analysis software (version 2.30, Nikon, Tokyo, Japan).

The two-dimensional digitized images were captured as TIFF files and imported into the morphometric software ImageJ, NIH. These laser scanning confocal images have been manipulated in Photoshop to adjust color values, remove artifacts, and increase sharpness. For each RLM (repair and control side), the total perimeter of the cell body outline was estimated and the total extension of the sections of the outlined circumference occupied by synaptophysin-immunoreactive profiles was measured. The ratio occupied by the labeled structure of interest was then calculated, the mean value for each specimen was also calculated and the percentage of repaired versus control side was generated, followed by quantitative comparisons (\%) between the two repair methods.

\section{LGC WEIGHT}

The LGC of the repaired and control sides of each animal was dissected free from its origin and insertion and weighed immediately while still wet at the time of euthanasia. LGC weight data were calculated as percentages of those for the contralateral unaffected limb of the same rat to correct for individual differences.

\section{STATISTICAL ANALYSIS}

The statistical analysis was performed using one-way ANOVA, followed by Student's $t$-test for counts comparing the two repair groups. All statistical tests were performed using the software GraphPad Prism 4.0 (GraphPad Software, Inc., San Diego, CA, USA). Statistical significance was set at $P<0.05$.

\section{RESULTS}

During the first postoperative month one rat from the adhesive group was killed because of a severe wound infection and one rat from the suture group was likewise killed because of clinical signs of far advanced self-harm.

\section{FLUORESCENCE MICROSCOPY AND MORPHOLOGICAL EXAMINATION}

Cell bodies labeled with CTB antibodies and/or CGRP antibodies were observed bilaterally in each of the 16 experimental rats and 
Table 1 | Mean (SD) morphometric data on cross-sectional distribution area $\left(\mu \mathrm{m}^{2} \times 10^{3}\right)$ and correlation (\%) between repair and control, respectively, of RLMs representing the LGC in the lateral ventral horn throughout the $\mathbf{L} 5$ spinal cord segment $\mathbf{6}$ months after transection and repair of the left rat sciatic nerve with either ECA or epineurial sutures ( $n=8$ in each group).

\begin{tabular}{llll}
\hline & Suture & ECA & $P$-value \\
\hline Repair & $340(53)$ & $317(58)$ & \\
Control & $148(22)$ & $155(40)$ & \\
Repair/ctrl \% & $232(45)$ & $210(35)$ & 0.29
\end{tabular}

were easily distinguished from unlabeled cells located in the gray matter of the lateral ventral horn extending through L5(Figure 2).

\section{RETROGRADE TRACING OF REGENERATED MOTONEURON}

Quantitative data showed a slightly more than a doubling of the anatomical cross-sectional distribution area of RLMs at the spinal L5 level in the suture group, compared to controls, whereas this increase were slightly less for the adhesions. Nevertheless, no significant differences were demonstrated (Table 1). The number of detected RLMs representing the LGC, located in the lateral ventral horn of the L5 spinal cord, was increased for both repair methods compared to the control side. No statistical difference was seen between the two repair methods (Figure 3). Without any detectable difference between the two repair methods, the number of RLMs located within the assumed normal anatomical distribution area in the gray matter was slightly more than half of the total number of RLMs distributed on the repair side. While the mean number of RLMs within the assumed normal myotopic area was slightly larger in the group repaired with ECA than in the sutured group, this difference was not significant (Figure 3).

In most controls, the RLMs appeared to merge so as to form one common column that occupied the described dorso-medial position in the lateral ventral horn of the gray matter. RLMs that were situated beyond the assumed normal myotopic area on the repair side could not be distinguished from corresponding profiles within this area or controls with respect to cell body shape, size (not shown), fluorescence intensity, or neurite appearance.

The measured mean cross-sectional area of RLMs in the lateral ventral horn was similar for the two repair methods but was somewhat increased compared to controls, although not significantly so (Table 2).

\section{DOUBLE-LABELING}

Retrogradely labeled motoneurons were assessed in the approximate ratio of 1 to 3 in relation to CGRP-labeled motoneurons (sciatic nerve-mediated neurons) in the lateral ventral horn irrespective of the repair method. A corresponding assessment of the contralateral control side showed an approximate ratio of 1 to 4 . RLMs in all sections were also consistently found to be CGRPpositive when appropriate fluorophores and filter combinations for double-labeling were utilized. The ratio of CGRP-labeled motoneurons from the ipsilateral repair side and the contralateral

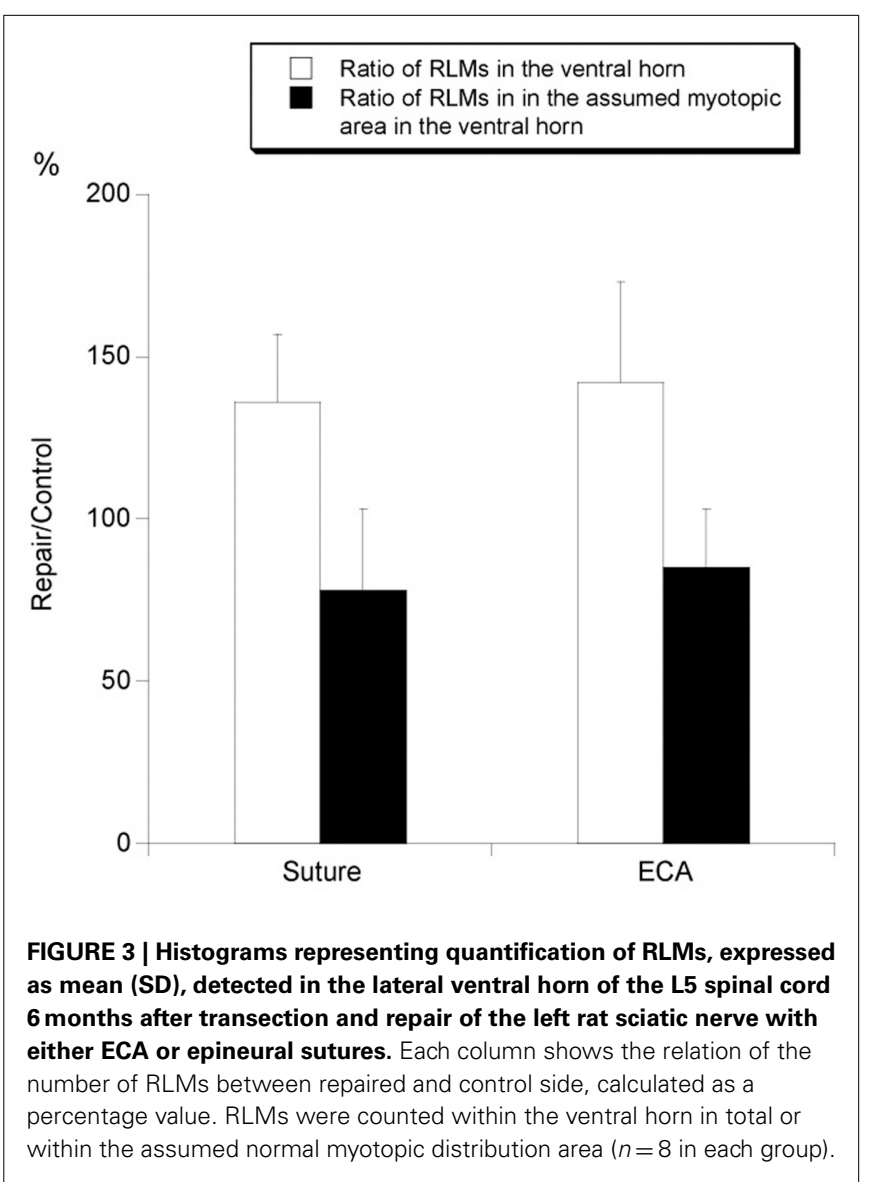

Table 2 | Mean (SD) morphometric data on cross-sectional soma size area $\left(\mu \mathrm{m}^{2}\right)$ and correlation (\%) between repair and control, respectively, of RLMs representing the LGC in the lateral ventral horn of the L5 spinal cord 6 months after transection and repair of the left rat sciatic nerve with either ECA or epineurial sutures ( $n=8$ in each group).

\begin{tabular}{llll}
\hline & Suture & ECA & $P$-value \\
\hline Repair & $2210(183)$ & $2234(80)$ & \\
Control & $2136(137)$ & $2057(153)$ & \\
Repair/ctrl \% & $103(12)$ & $109(7)$ & 0.31
\end{tabular}

control side was calculated to be approximately $89 \%(\mathrm{SD} \pm 5 \%$ ) in the ECA repair group and $91 \%(\mathrm{SD} \pm 8 \%)$ in the suture repair group. Using fluorescence microscopy, the boundaries of cell bodies and proximal dendrites of RLMs, with a negligible number of exceptions, were found to be covered by synaptophysinimmunoreactive profiles. The major portion of the circumference was found to be coated by these profiles, although with an irregular degree of fluorescence intensity and a rough appearance along the outline (Figure 4). No difference in the extent of labeling or fluorescence intensity of immunoreactivity for synaptophysin between the two repair methods, or compared to controls, could be revealed by fluorescence microscopy. 

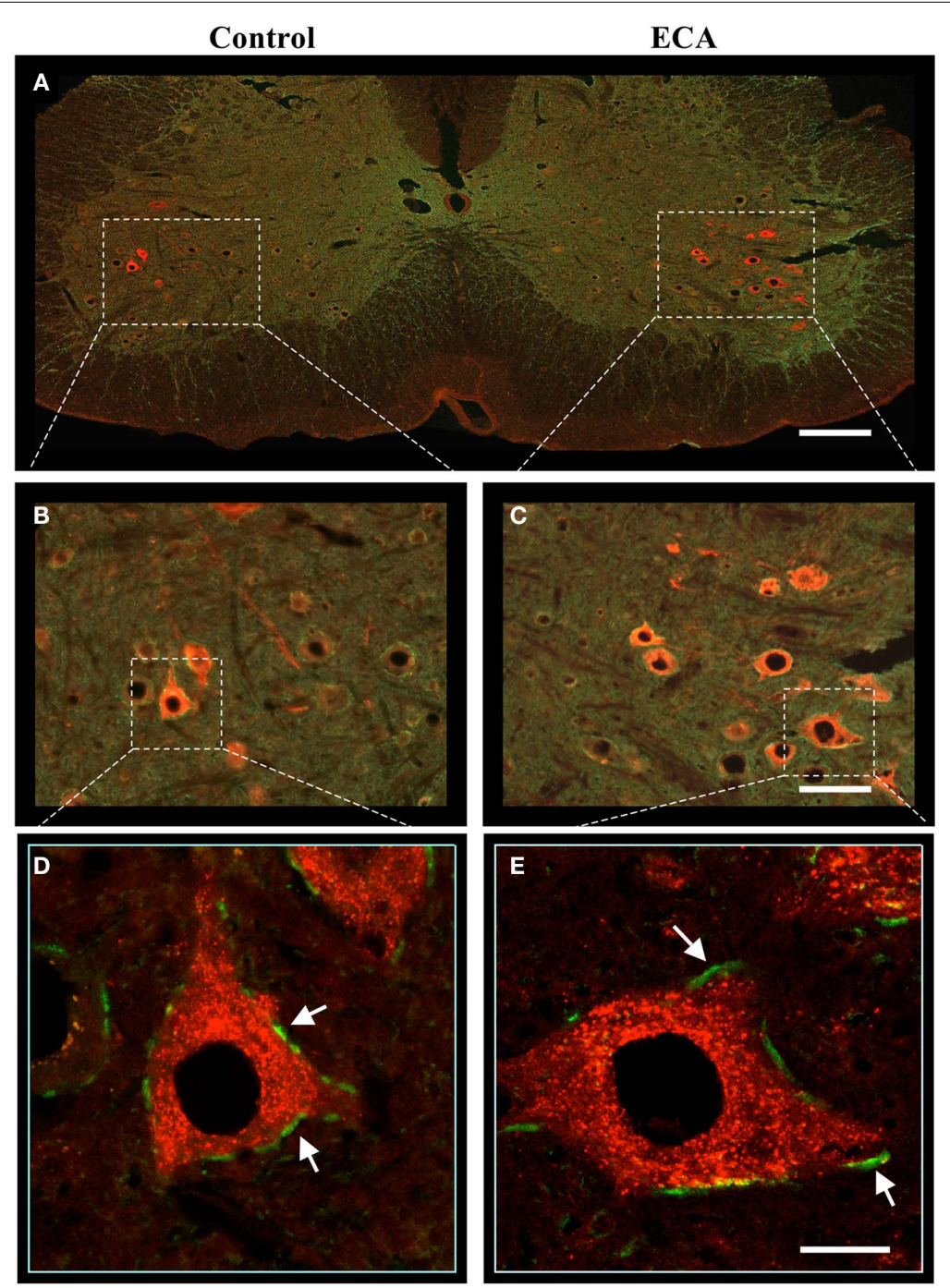

FIGURE 4 | Transverse L5 spinal cord section 6 months after transection and repair of the left rat sciatic nerve with ETC and double-labeling with anti-synaptophysin (green) and anti-CTB (red). Microphotographs showing RLMs in the lateral ventral horn from the ipsilateral ECA-repaired side $(\mathbf{A}, \mathbf{C})$ and the uninjured contralateral side $(\mathbf{A}, \mathbf{B})$. Note the greater number and the scattered distribution of the RLMs on the repaired side. Confocal projection images (D,E) showing contacts (arrows) between synaptophysin immuno-labeled structures (displayed in green) along the membrane of the RLMs (displayed in red). Immunoreactivity for synaptophysin in the assessed area was consistently reduced in injured motoneurons but did not reveal any significant difference between the two repair methods. Scale bar (A) $=250 \mu \mathrm{m},(\mathbf{B}, \mathbf{C})=100 \mu \mathrm{m},(\mathbf{D}, \mathbf{E})=25 \mu \mathrm{m}$

\section{CONFOCAL MICROSCOPY FOR SYNAPTOPHYSIN-IMMUNOREACTIVE PROFILES}

Confocal microscopy further confirmed the apposition of synaptophysin-immunoreactive profiles on $\alpha-\mathrm{MNs}$, on both their cell bodies and proximal dendrites (Figures 4D,E). A quantitative estimation indicated some reduction after injury, irrespective of the repair method, when repaired versus control sides was correlated.

Immunoreactivity for synaptophysin over the ECA-repaired motoneuron pool was somewhat lower compared to the corresponding ratio for the suture-repaired motoneuron pool. When ECA and suture repair were compared, no significant difference in synaptophysin expression could be seen (Table 3).
Table 3 Mean (SD) morphometric data on the degree of intensity of synaptophysin-labeled profiles in contact with RLMs in the lateral ventral horn of the $\mathbf{L} 5$ spinal cord $\mathbf{6}$ months after transection and repair of the left rat sciatic nerve with either ECA or epineurial sutures. The ratio occupied by the labeled structure of interest was estimated as the percentage $(\%)$ of the repaired versus the control side ( $n=6$ in each group).

\begin{tabular}{llll}
\hline & Suture & ECA & $P$-value \\
\hline Repair & $23(1)$ & $24(2)$ & \\
Control & $29(4)$ & $31(3)$ & \\
Repair/ctrl \% & $79(12)$ & $76(9)$ & 0.47
\end{tabular}




\section{LATERAL GASTROCNEMIUS MUSCLE WEIGHT}

On visual examination, the LGCs on the operated sides demonstrated noticeable atrophy compared to the contralateral normal limbs. This could also be demonstrated by reduced muscle weight on the experimental side, although no statistical difference could be detected between the two repair methods (Figure 5).

\section{DISCUSSION}

This study demonstrates that the precision in reinnervation of the LGC is far from perfect after repair with ECA. However, compared to conventional microsutures, the results appear to be comparable. Thus, no significant difference was seen with regard to localization in the ventral horn or the number, size or synaptic covering of reinnervating $\alpha$-MNs between the two repair methods.

In the present study we used retrograde tracing as a tool to assess the accuracy of reinnervation after repair with ECA. The selectivity of reinnervation of the LGC was studied. The response of the LGC is well characterized from animal studies and it is mainly innervated at spinal level L5 (Tredici et al., 1996). The tracer was administered bilaterally to the tibial nerve branch close to the entrance into the LGC in order to detect motoneurons that had innervated this nerve branch and to evaluate the threedimensional distribution of its spinal cord motor nuclei in order to document the alterations of the spinal myotopy due to peripheral reinnervation after repair using two different methods. There are a few methodological considerations connected with the technique employed, such as leakage or uptake in the soleus nerve branch (Leong and Ling, 1990). It seems more difficult to achieve a complete and reproducible uptake from intramuscular injections, which is probably related to the fact that this muscle is composed

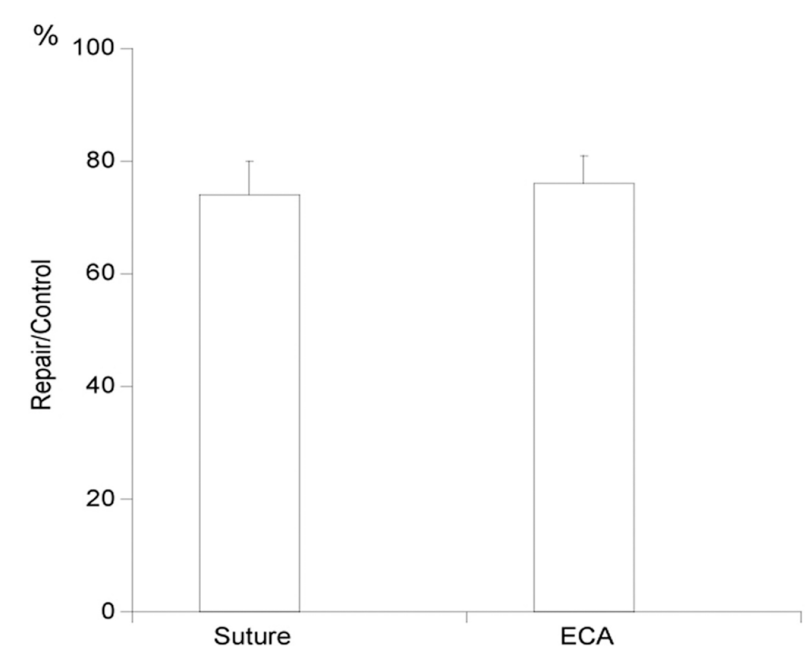

FIGURE 5 | Histogram representing the wet weight, expressed as mean (SD), of the lateral gastrocnemius muscles (LGCs) 6 months after transection and repair of the left sciatic nerve with either ECA or epineurial sutures. Each column shows the weight relationship (\%) of the LGCs calculated by dividing the muscle mass of the repaired side by the muscle mass of the control side ( $n=8$ in each group). of four subdivisions. However, the methodological problems and limitations should be similar in both examined repair methods.

The outcome of nerve repair can be assumed to be the result of several factors, such as retrograde degeneration of injured neurons and the effectiveness and accuracy of reinnervation. Synaptic plasticity in the spinal cord is probably also a limiting factor. Most studies indicate that the retrograde loss of motoneurons is very limited in adult animals (Arvidsson and Aldskogius, 1982; Schmalbruch, 1984), whereas sensory, a substantial proportion of the injured dorsal root ganglion neurons may be lost (Aldskogius et al., 1985). Furthermore, return of sensory function can be a limiting factor for the functional outcome. Additionally, in this study we have concentrated on acute repair. The outcome after a delayed repair can be assumed to be worse and could possibly differ between the methods, as an additional resection of the injured nerve stumps is usually necessary.

Two prevailing views have emerged about how nerve sprouts could find the correct route to their original target muscle. One is that the Schwann cell tubes of the respective pathways maintain a specific, interpreted identity that can be recognized by regenerating motor axons. The other opinion is that regenerating motor axons (randomly) assess the relative levels of trophic support in each pathway and preferentially remain in the one that provides the greater amount of support, so-called "pruning" (i.e., the withdrawal of the axon branches that reinnervate the wrong target; see detailed review: Madison et al., 2007). In this study we have assessed, by using retrograde tracing techniques, whether a synthetic adhesive, added to the lesioned nerve ending prior to coaptation, would exert any negative effect on the accuracy of the regrowth. In our present study, motoneurons projecting to a specific muscle were scattered throughout a larger volume of the spinal cord gray matter than in controls and were intermingled within the lumbar ventral horn, suggesting that motoneurons originally belonging to adjacent nuclei aberrantly project to the experimental muscle after regeneration. Valero-Cabre et al. (2004) demonstrated a spreading of the tibialis anterior nucleus to more ventral regions of the spinal cord gray matter, indicating that motoneurons originally projecting to the LGC aberrantly reinnervate the tibialis anterior muscle after nerve cut and repair, and vice versa. Our quantitative results show an increase in the proportion of transversal expansion of the LGC. These results are consistent with Valero-Cabre et al. (2004) who found three motor nuclei from the hind limb significantly enlarged in the transversal perimeter after sciatic nerve transection and suture repair. The most marked increase was in the anterior-posterior axis of the nucleus of the LGC. Our findings of an apparent increase in the number, together with a larger myotopic distribution area, of RLMs, regardless of the repair method, can be seen consistently in previous reports. However, regenerating axons have been shown to form more than one branch (Shawe, 1955) and, as a consequence, polyinnervation, i.e., the attachment endplate of more than one motoneuron to the same endplate (Ijkema-Paassen et al., 2002), hyperinnervation, i.e., the projection of more motoneurons into the same muscle after reinnervation than before lesion (Angelov et al., 1993) and axonal trunk bifurcation, i.e., simultaneous axonal branches to more than one muscle from long collateral branches of the same motoneuron (Hennig and Dietrichs, 1994), all being contributory factors 
that might largely explain the increase in number as well as the enlarged and disorganized myotopic distribution of RLMs once reinnervation had been completed.

Within the assumed normal myotopic distribution area we found that the number of RLMs was reduced, regardless of the repair method, by approximately one-fourth on the repair side compared to the control side. The number of labeled neurons is therefore an indication of surviving cells reinnervating the motor targets, and a reduction after repair may be attributed to axonal misdirection to non-labeled targets rather than to neuronal death. In any case, with a more sparse $\alpha-M N$ population in the assumed normal myotopic distribution area connected to the original peripheral target, there follows a presumed decrease in appropriate supraspinal and propriospinal input, resulting in reduced functionality in the muscle.

Retraction of axonal terminals from the surface of injured motoneurons is a well-known phenomenon. A preferential reduction of excitatory synapses could possibly have a neuroprotective role (Linda et al., 1992). Brannstrom and Kellerth (1998) studied the ultrastructural changes in the synaptology of adult cat spinal $\alpha-\mathrm{MNs}$ after axotomy of the medial gastrocnemius nerve. At 12 weeks after permanent axotomy, the synaptic covering was reduced by $83 \%$ on the cell soma compared to the normal situation. In a parallel study by the same authors, the nerve was allowed to reinnervate its muscle through a nerve graft after 6 weeks of ligation. Two years later, the axotomized and reinnervated motoneurons had not regained their original synaptic covering, which was still reduced by more than $25 \%$ as compared to the normal situation (Brannstrom et al., 1992).

Synaptophysin, used in our study, is a presynaptic membrane protein of neurotransmitter-containing synaptic vesicles and is expressed ubiquitously throughout all synapses of the CNS and PNS (Wiedenmann and Franke, 1985). Synaptophysin immunostaining is thus a good marker for presynaptic terminals which has been widely used to estimate the increase or decrease in the number of synapses on identified motoneurons (Zang et al., 2005). In both of our experimental repair groups we found a similar synaptic coverage of labeled motoneurons, although a significant decrease compared to control sides of approximately one-fifth. These findings suggest that the existing reinnervated $\alpha$-MNs in the LGC nucleus, 6 months after transection and repair of the sciatic nerve, are subjected to an additive reduction (compared to the control side) in synaptic inputs which could result in a further reduction in muscle functionality.

From a clinical perspective, complementary functional tests (i.e., walking pattern, foot withdrawal reflex or toe spreading reflex tests) could be desirable to increase our understanding of the fate of motoneurons after surgical nerve repair with a synthetic adhesive. However, Beer et al. (2008) showed muscle weight to be a more detailed method to evaluate functional recovery than toe spreading reflex testing and thereby justify its use as a functional parameter of motor regeneration. In our present study, an obvious reduction in weight of the LGC, compared to controls, but without any difference between the two repair methods, was observed 6 months after repair. One limitation is that by measuring the muscle weight alone, only the weight, but not the strength, of the muscle is measured.

\section{CONCLUSION}

Previous studies have suggested that for peripheral nerve repair in the rat, there is no difference between synthetic adhesive and microsutures with respect to recovery of function or electrophysiological and morphometric indices of recovery in the peripheral nerve (Choi et al., 2004; Pineros-Fernandez et al., 2005; Landegren et al., 2006). These studies have, however not looked upon the morphological selectivity of regeneration. The limited number of animals used in this study shows a tendency to greater labeling of anterior horn cells from the LGC when synthetic adhesives are used, but this finding does not reach statistical significance. A larger study involving more animals might reveal whether this difference is significant.

Moreover, this study has involved immediate repair only. Despite an increasing amount of information showing that delayed repair is inferior to immediate repair (Bignotti et al., 1986), the former is widely used for practical reasons in the clinical situation. It would be of considerable interest to compare the results obtained here with a clinically relevant experimental study in which delayed repair of the peripheral nerve was used. It seems likely, from a speculative point of view, that this avenue will prove to be fruitful in distinguishing the relative values of immediate and late repair of peripheral nerves.

\section{ACKNOWLEDGMENTS}

The present study was supported by the Stockholm County Council, the Swedish Defence Research Agency Innovation Foundation, and the Karolinska Institutet, Stockholm, Sweden. The excellent technical assistance given by Ms. Maria Angeria and MS. Birgitta Robertsson is gratefully acknowledged. We thank Peter Rosén, Pixmix, for his indispensable help in the photographic artwork and graphics processing.

\section{REFERENCES}

Aldskogius, H., Arvidsson, J., and Grant, G. (1985). The reaction of primary sensory neurons to peripheral nerve injury with particular emphasis on transganglionic changes. Brain Res. 357, 27-46.

Angelov, D. N., Gunkel, A., Stennert, E., and Neiss, W. F. (1993). Recovery of original nerve supply after hypoglossal-facial anastomosis causes permanent motor hyperinnervation of the whisker-pad muscles in the rat. J. Comp. Neurol. 338, 214-224.

Arvidsson, J., and Aldskogius, H. (1982). Effect of repeated hypoglossal nerve lesions on the number of neurons in the hypoglossal nucleus of adult rats. Exp. Neurol. 75, 520-524.
Beer, G. M., Seifert, B., Schneller, M., Iscru, G., and Schmitz, H. C. (2008). Relevance of muscle weight as a functional parameter for the regeneration of the peroneal nerve in rabbits. J. Reconstr. Microsurg. 24, 11-19.

Bertelli, J. A., and Mira, J. C. (1993). Nerve repair using freezing and fibrin glue: immediate histologic improvement of axonal coaptation. Microsurgery 14, 135-140.

Bignotti, B., Origo, C., Schenone, A., Ratto, S., Mancardi, G. L., and Ferrari, M. L. (1986). Experimental studies on peripheral nerve repair following early or delayed suture. Ital. J. Orthop. Traumatol. 12, 259-266. 
Brannstrom, T., Havton, L., and Kellerth, J. O. (1992). Restorative effects of reinnervation on the size and dendritic arborization patterns of axotomized cat spinal alphamotoneurons. J. Comp. Neurol. 318, 452-461.

Brannstrom, T., and Kellerth, J. O. (1998). Changes in synaptology of adult cat spinal alpha-motoneurons after axotomy. Exp. Brain Res. 118, 1-13.

Brushart, T. M. (1993). Motor axons preferentially reinnervate motor pathways. J. Neurosci. 13, 2730-2738.

Brushart, T. M., Tarlov, E. C., and Mesulam, M. M. (1983). Specificity of muscle reinnervation after epineurial and individual fascicular suture of the rat sciatic nerve. J. Hand. Surg. Am. 8, 248-253.

Choi, B. H., Kim, B. Y., Huh, J. Y., Lee, S. H., Zhu, S. J., Jung, J. H., and Cho, B. P. (2004). Microneural anastomosis using cyanoacrylate adhesives. Int. J. Oral Maxillofac. Surg. 33, 777-780.

Dvali, L., and Mackinnon, S. (2007). The role of microsurgery in nerve repair and nerve grafting. Hand Clin. 23, 73-81.

Fu, S. Y., and Gordon, T. (1995a). Contributing factors to poor functional recovery after delayed nerve repair: prolonged denervation. J. Neurosci. 15(Pt 2), 3886-3895.

Fu, S. Y., and Gordon, T. (1995b). Contributing factors to poor functional recovery after delayed nerve repair: prolonged axotomy. J. Neurosci. 15(Pt 2), 3876-3885.

Galtrey, C. M., and Fawcett, J. W. (2007). Characterization of tests of functional recovery after median and ulnar nerve injury and repair in the rat forelimb. J. Peripher. Nerv. Syst. 12, 11-27.
Hennig, R., and Dietrichs, E. (1994). Transient reinnervation of antagonistic muscles by the same motoneuron. Exp. Neurol. 130, 331-336.

Hoke, A., Redett, R., Hameed, H., Jari, R., Zhou, C., Li, Z. B., Griffin, J. W., and Brushart, T. M. (2006). Schwann cells express motor and sensory phenotypes that regulate axon regeneration. J. Neurosci. 26, 9646-9655.

Ijkema-Paassen, J., Meek, M. F., and Gramsbergen, A. (2002). Reinnervation of muscles after transection of the sciatic nerve in adult rats. Muscle Nerve 25, 891-897.

Landegren, T., Risling, M., Brage, A., and Persson, J. K. (2006). Long-term results of peripheral nerve repair: a comparison of nerve anastomosis with ethyl-cyanoacrylate and epineural sutures. Scand. J. Plast. Reconstr. Surg. Hand. Surg. 40, 65-72.

Leong, S. K., and Ling, E. A. (1990). Labelling neurons with fluorescent dyes administered via intravenous, subcutaneous or intraperitoneal route. J. Neurosci. Methods 32, 15-23.

Linda, H., Cullheim, S., and Risling, M. (1992). A light and electron microscopic study of intracellularly HRP-labeled lumbar motoneurons after intramedullary axotomy in the adult cat. J. Comp. Neurol. 318, 188-208.

Madison, R. D., Robinson, G. A., and Chadaram, S. R. (2007). The specificity of motor neurone regeneration (preferential reinnervation). Acta Physiol. (Oxf.) 189, 201-206.

Millesi, H. (1973). Microsurgery of peripheral nerves. Hand 5, 157-160.

Pineros-Fernandez, A., Rodeheaver, P. F., and Rodeheaver, G. T. (2005). Octyl 2-cyanoacrylate for repair of peripheral nerve. Ann. Plast. Surg. 55, 188-195.
Schmalbruch, H. (1984). Motoneuron death after sciatic nerve section in newborn rats. J. Comp. Neurol. 224 252-258.

Shawe, G. D. (1955). On the number of branches formed by regenerating nerve-fibres. Br. J. Surg. 42, 474-488.

Siemionow, M., and Brzezicki, G. (2009). Chapter 8: current techniques and concepts in peripheral nerve repair. Int. Rev. Neurobiol. 87, 141-172.

Sullivan, D. J. (1985). Results of digital neurorrhaphy in adults. J. Hand Surg. Br. 10, 41-44.

Sumner, A. J. (1990). Aberrant reinnervation. Muscle Nerve 13, 801-803.

Suri, A., Mehta, V. S., and Sarkar, C. (2002). Microneural anastomosis with fibrin glue: an experimental study. Neurol. India 50, 23-26.

Tredici, G., Migliorini, C., Barajon, I., Cavaletti, G., and Cece, R. (1996). Anatomical organization of the spinal paths to the soleus and gastrocnemius muscles of the rat hind limb. J. Hirnforsch. 37, 81-89.

Valero-Cabre, A., Tsironis, K., Skouras, E., Navarro, X., and Neiss, W. F. (2004). Peripheral and spinal motor reorganization after nerve injury and repair. J. Neurotrauma 21 , 95-108.

Verdu, E., Ceballos, D., Vilches, J. J. and Navarro, X. (2000). Influence of aging on peripheral nerve function and regeneration. J. Peripher. Nerv. Syst. 5, 191-208.

Vertruyen, M. F., Burgeon, M. A., Dachy, B. S., and Ley, R. E. (1994). Sensory recovery after microsurgical repair of digital nerves. Acta Chir. Belg. 94 325-328.

Wiedenmann, B., and Franke, W. W. (1985). Identification and localization of synaptophysin, an integral membrane glycoprotein of $\mathrm{Mr}$
38,000 characteristic of presynaptic vesicles. Cell 41, 1017-1028.

Zang, D. W., Lopes, E. C., and Cheema, S. S. (2005). Loss of synaptophysin-positive boutons on lumbar motor neurons innervating the medial gastrocnemius muscle of the SOD1G93A G1H transgenic mouse model of ALS. J. Neurosci. Res. 79, 694-699.

Zhao, Q., Dahlin, L. B., Kanje, M. and Lundborg, G. (1992). Specificity of muscle reinnervation following repair of the transected sciatic nerve. A comparative study of different repair techniques in the rat. J. Hand Surg. Br. 17, 257-261.

Conflict of Interest Statement: The authors declare that the research was conducted in the absence of any commercial or financial relationships that could be construed as a potential conflict of interest.

Received: 03 December 2010; paper pending published: 01 February 2011; accepted: 03 April 2011; published online: 27 April 2011.

Citation: Landegren T, Risling M, Hammarberg $H$ and Persson JKE (2011) Selectivity in the reinnervation of the lateral gastrocnemius muscle after nerve repair with ethyl cyanoacrylate in the rat. Front. Neur. 2:25. doi: 10.3389/fneur.2011.00025

This article was submitted to Frontiers in Neurotrauma, a specialty of Frontiers in Neurology.

Copyright (C) 2011 Landegren, Risling, Hammarberg and Persson. This is an open-access article subject to a nonexclusive license between the authors and Frontiers Media SA, which permits use, distribution and reproduction in other forums, provided the original authors and source are credited and other Frontiers conditions are complied with. 